\title{
Two Heads Are Better than Two Tapes
}

\author{
TAO JIANG \\ McMaster University, Hamilton, Ontario, Canada
}

JOEL I. SEIFERAS

University of Rochester, Rochester, New York

\begin{abstract}
AND
PAUL M. B. VITÁNYI

CWI and Universiteit van Amsterdam, Amsterdam, The Netherlands
\end{abstract}

\begin{abstract}
We show that a Turing machine with two single-head one-dimensional tapes cannot recognize the set

$$
\left\{x 2 x^{\prime} \mid x \in\{0,1\}^{*} \text { and } x^{\prime} \text { is a prefix of } x\right\}
$$

in real time, although it can do so with three tapes, two two-dimensional tapes, or one two-head tape, or in linear time with just one tape. In particular, this settles the longstanding conjecture that a two-head Turing machine can recognize more languages in real time if its heads are on the same one-dimensional tape than if they are on separate one-dimensional tapes.

Categories and Subject Descriptors: E.2 [Data]: Data Storage Representations; F.1.1 [Computation by Abstract Devices]: Models of Computation-relations among models, bounded-action devices, automata; F.2.3 [Analysis of Algorithms and Problem Complexity]: Tradeoffs among Complexity Measures; F.2.2 [Analysis of Algorithms and Problem Complexity]: Nonnumerical Algorithms and Problems-pattern matching; G.2.m [Discrete Mathematics]: Miscellaneous
\end{abstract}

General Terms: Theory

The work of T. Jiang was supported in part by NSERC Operating Grant OGP0046613. The work of P. M. B. Vitányi was supported in part by the European Union through NeuroCOLT ESPRIT Working Group Number 8556, and by NWO through NFI Project ALADDIN under Contract Number NF 62-376.

An earlier version of this report appeared in Proceedings of the 26th Annual ACM Symposium on the Theory of Computing (Montreal, Que., Canada, May 23-25). ACM, New York, 1994, pp. 668-675.

Authors' addresses: T. Jiang, Department of Computer Science, McMaster University, Hamilton, Ontario L8S 4K1, Canada, e-mail: jiang@maccs.mcmaster.ca; J. I. Seiferas, Computer Science Department, University of Rochester, Rochester, NY 14627-0226, e-mail: joel@cs.rochester.edu; P. M. B. Vitányi, Centre for Mathematics and Computer Science (CWI), Kruislaan 413, 1098 SJ Amsterdam, The Netherlands, e-mail: paulv@cwi.nl.

Permission to make digital/hard copy of part or all of this work for personal or classroom use is granted without fee provided that the copies are not made or distributed for profit or commercial advantage, the copyright notice, the title of the publication, and its date appear, and notice is given that copying is by permission of the Association for Computing Machinery (ACM), Inc. To copy otherwise, to republish, to post on servers, or to redistribute to lists, requires prior specific permission and/or a fee.

(C) 1997 ACM 0004-5411/97/0300-0237 \$03.50 
Additional Key Words and Phrases: Buffer, heads vs. tapes, Kolmogorov complexity, lower bound, multihead tape, multitape Turing machine, on-line simulation, overlap, queue, real-time simulation, single-head tapes, two-head tape

\section{Introduction}

The Turing machines commonly used and studied in computer science have separate tapes for input/output and for storage, so that we can conveniently study both storage as a dynamic resource and the more complex storage structures required for efficient implementation of practical algorithms [Hartmanis and Stearns 1965]. Early researchers [Meyer et al. 1967] asked specifically whether two-head storage is more powerful if both heads are on the same one-dimensional storage tape than if they are on separate one-dimensional tapes, an issue of whether shared sequential storage is more powerful than separate sequential storage. Our result settles the longstanding conjecture that it is.

In a broader context, there are several natural structural parameters for the storage tapes of a Turing machine. These include the number of tapes, the dimension of the tapes, and the number of heads on each tape. It is natural to conjecture that a deficiency in any such parameter is significant and cannot be fully compensated for by advantages in the others. For the most part, this has indeed turned out to be the case, although the proofs have been disproportionately difficult. ${ }^{1}$

The case of deficiency in the number of heads allowed on each tape has turned out to be the most delicate, because it involves a surprise: A larger number of single-head tapes can compensate for the absence of multihead tapes [Meyer et al. 1967; Fischer et al. 1972; Leong and Seiferas 1981]. For example, four single-head tapes suffice for general simulation of a two-head tape unit, without any time loss at all [Leong and Seiferas 1981]. The remaining question is just what, if anything, is the advantage of multihead tapes.

The simplest version of the question is whether a two-head tape is more powerful than two single-head tapes. In the case of "tapes" that are multidimensional, Paul has shown that it is [Paul 1984]. His proof involves using the two-head tape to write, and occasionally to retrieve parts of, algorithmically incompressible bit patterns. Because the diameter of a multidimensional pattern (and hence the retrieval times) can be kept much smaller than its volume, no fast simulator would ever have time to perform any significant revision or copying of its representation of the bit pattern. On ordinary one-dimensional tapes, however, retrievals take time that is not small compared to the volume of data, and we cannot so easily focus on a nearly static representation of the data. We need some more subtle way to rule out all (possibly very obscure) copying methods that a two-tape machine might employ to keep up with its mission of fast simulation. Our argument below does finally get a handle on this elusive "copying" issue, making use of a lemma formulated more than ten years ago with this goal already in mind [Vitányi 1984, Far-Out Lemma below].

\footnotetext{
${ }^{1}$ See, for example, Rabin [1963], Hennie [1966], Grigoriev [1977], Aanderaa [1974], Paul et al. [1981], Paul [1982], Duriš et al. [1984], Maass [1985], Li and Vitányi [1988], Li et al. [1992], Maass et al. [1993], and Paturi et al. [1990].
} 
Our specific result is that no Turing machine with just two single-head one-dimensional storage tapes can recognize the following language in real time: ${ }^{2}$

$$
L=\left\{x 2 x^{\prime} \mid x \in\{0,1\}^{*} \text { and } x^{\prime} \text { is a prefix of } x\right\} .
$$

With a two-head tape, a Turing machine can easily recognize $L$ in real time.

Our result incidentally gives us a tight bound on the number of single-head tapes needed to recognize the particular language $L$ in real time, since three do suffice [Meyer et al. 1967; Fischer et al. 1972]. Thus, $L$ is another example of a language with "number-of-tapes complexity" 3, rather different from the one first given by Aanderaa [Aanderaa 1974; Paul et al. 1981]. (For the latter, even a two-head tape, even if enhanced by instantaneous head-to-head jumps and allowed to operate probabilistically, was not enough [Paturi et al. 1990].)

Historically, multihead tapes were introduced in Hartmanis and Stearns' * seminal paper [Hartmanis and Stearns 1965] which outlined a linear-time ${ }^{2}$ simulation of an $h$-head tape, using some larger number of ordinary single-head tapes. Stoß [1970] later reduced the number of single-head tapes to just $h$. Noting the existence of an easy real-time simulation in the other direction, Bečvár [1965] explicitly raised the question of real-time simulation of an $h$-head tape using only single-head tapes. Meyer et al. [1967] devised the first such simulation; and others later reduced the number of tapes [Fischer et al. 1972; Bennison 1974; Leong and Seiferas 1981], ultimately to just $4 h-4$. We are the first to show that this number cannot always be reduced to just $h$, although both the extra power of multihead tapes and the more-than-two-tape complexity of the particular language $L$ have been longstanding conjectures. ${ }^{3}$

The reader may have noticed that we use, and even mix, two apparently separate terminologies, that of Turing machines and language recognition, on the one hand, and that of storage structures and simulations on the other. These are actually closely related, however, the choice being only one of emphasis.

The main components of a "Turing machine", of course, are a finite-state controller and some sort of simple storage (usually tape) of unlimited capacity. (We assume any input or output is through other, separate peripheral devices to which the controller has access. In our case of on-line language recognition, ${ }^{2}$ input symbols are consumed and output verdicts are produced sequentially, so that one-way input and output tapes would do.) When our focus is on the nature of the storage structure, we view it separately as a sequential machine (or "abstract storage unit") in its own right-one that responds in an on-line manner to input commands from some finite alphabet with appropriate output responses from some other finite alphabet. For some input commands, such as "report the symbol scanned by the second head", the precise response is significant; but, for

\footnotetext{
${ }^{2}$ On-line recognition requires a verdict for each input prefix before the next input symbol is read, and real-time recognition is on-line recognition with some constant delay bound on the number of steps between the reading of successive input symbols. Note that even a single-tape Turing machine can recognize $L$ on-line in cumulative linear time; but this involves an unbounded (linear-time) delay to "rewind" after reading the symbol 2. In cumulative linear time, in fact, general on-line simulation of a two-head one-dimensional tape is possible using just two single-head tapes [Stoß 1970]; so real time is a stronger notion of "without time loss". (There is an analogous linear-time simulation for two-dimensional tapes [Schnitzlein and Stoß 1989], but the question is open for higher dimensions.) ${ }^{3}$ See, for example, Fischer et al. [1972], Leong and Seiferas [1981], Vitányi [1984], and Paul [1984].
} 
others, such as "shift the first head left" or "write the symbol 1 with the second head", it amounts to a mere acknowledgment. The on-line requirement in this setting is just that each command's response must precede the next command. If a machine, possibly with a completely different storage structure of its own, efficiently produces the same output behavior, then it (or even its storage structure, if that is our emphasis) can be said to efficiently simulate the storage structure. The strongest time-efficiency goal is real time, requiring that there be some single bound $d$ on the time delay between command receipt and response.

Note that several abstract storage units can be combined into one. The composite command alphabet is a disjoint union of the individual command alphabets. Thus, for example, a pair of ordinary one-head one-dimensional tapes can be viewed as a single abstract storage unit that happens to have the same command and response alphabets as a two-head one-dimensional tape unit. (There is no command to our two-head unit to report whether the two head positions coincide. Such a command is easy to simulate, however, so the issue is not important.)

An abstract storage unit with sufficiently atomic commands needs only a binary response alphabet $\{0,1\}$. The command to "report the symbol scanned by the second head", for example, can be replaced by some constant number of binary commands of the form "report whether the symbol scanned by the second head is $a$ ". Simulation of such a storage unit amounts to what is usually called an on-line language recognition problem (recall footnote 2), with 1 signalling "acceptance so far" and 0 signalling "rejection so far". It follows, for example, that a pair of single-head tapes can simulate a two-head tape in real time if and only if every language recognized in real time by a Turing machine with a two-head tape ("two-head Turing machine", for short) can be recognized in real time by a Turing machine with two single-head tapes ("two-tape Turing machine", for short), an issue that we resolve negatively in this paper.

\section{Tools}

2.1. Overlap. Part of our strategy will be to find within any computation a sufficiently long subcomputation that is sufficiently well behaved for the rest of our analysis. The behavior we seek involves limitations on repeated access to storage locations, which we call "overlap" [Aanderaa 1974; Paturi et al. 1990].

Our overlap lemma is purely combinatorial, and does not depend at all on the nature of our computations or the "storage locations" corresponding to their steps. Nor does it depend on the computational significance of the steps designated as "distinguished." The use of computational terminology would only obscure the lemma's formulation and proof, so we avoid it.

An overlap event in a sequence $S=\ell_{1}, \ldots, \ell_{T}$ (of "storage locations", in our application) is a pair $(i, j)$ of indices with $1 \leq i<j \leq T$ and $\ell_{i}=\ell_{j} \notin$ $\left\{\ell_{i+1}, \ldots, \ell_{j-1}\right\}$ (i.e., "visit and soonest revisit"). If $\omega_{t}(S)$ denotes the number of such overlap events "straddling" $t$ (i.e., with $i \leq t$ but $j \notin t$ ), then the sequence's internal overlap, $\omega(S)$, is $\max \left\{\omega_{t}(S) \mid 1 \leq t<T\right\}$. The relative internal overlap is $\omega(S) / T$.

Here is an example: In the sequence

$$
S=\text { cow, pig, horse, pig, sheep, horse, pig, }
$$


the overlap events are $(2,4),(4,7)$, and $(3,6)$. For $t$ from 1 up to 6 , the respective values of $\omega_{t}(S)$ are $0,1,2,2,2$, and 1 ; so $\omega(S)$ is 2 , and the relative internal overlap is $2 / 7$.

(In our setting below, we apply these definitions to the sequence of storage locations shifted to on the successive steps of a computation or subcomputation. Without loss of generality, we assume that a multihead or multitape machine shifts exactly one head on each step.)

The lemma we now formulate guarantees the existence of a contiguous subsequence that has "small" relative internal overlap (quantified using $\epsilon$ ), but that is itself still "long" (quantified using $\epsilon^{\prime}$ ). The lemma additionally guarantees that the subsequence can include a quite fair share of a set of "distinguished positions" of our choice in the original sequence.

(Without the latter guarantee, the lemma is just a simple corollary (and the essence) of any of the "overlap lemmas" formulated in the work we have already cited [Aanderaa 1974; Paturi et al. 1990]. The "designated positions" in our setting will be the items in the sequence that correspond to a large "matching"-a notion we define later, especially motivated by computations involving two heads.)

Overlap LemMA. Consider any $\delta<1$ and any $\epsilon>0$. Every sequence $S$ (of length $T$, say) with "distinguished-position" density at least $\delta$ has a long contiguous subsequence, of length at least $\epsilon^{\prime} T$ for some constant $\epsilon^{\prime}>0$ that depends only on $\delta$ and $\epsilon$, with distinguished-position density still at least $\delta / 4$, and with relative internal overlap less than $\epsilon$.

Proof. Without loss of generality, assume $T$ is large in terms of $\delta$ and $\epsilon$. It suffices to assume $T$ is a power of 2 , and to aim for a subsequence with distinguished-position density still at least $\delta / 2$ (better than our general goal of $\delta / 4$ ). (If the given sequence does not have length that is a power of 2 , then we can discard an appropriate prefix or suffix of length less than half the total length, to obtain such a sequence with distinguished-position density still at least $\delta / 2$, and then work with that sequence instead.) We consider only the sequence's two halves, four quarters, eight eighths, etc. Of these, we seek many with sufficient distinguished-position density (at least $\delta / 2$ ) and with internal overlap accounted for by distinct overlap events, planning then to use the fact that each item in $S$ can serve as the second component of at most one overlap event.

Within each candidate subsequence $S^{\prime}$, we can select a particular straddle point $t$ for which $\omega\left(S^{\prime}\right)=\omega_{t}\left(S^{\prime}\right)$, and then we can designate the $\omega\left(S^{\prime}\right)$ overlap events within $S^{\prime}$ that straddle position $t$ as the ones we consider counting. The designated overlap events in $S^{\prime}$ can be shared by another interval only if that interval includes the corresponding selected straddle point $t$.

We consider the candidate sequences in order of decreasing length (i.e., halves, then quarters, then eighths, etc.). At each partitioning level, at least fraction $\delta / 2$ of the subsequences must have distinguished-position density at least $\delta / 2$. (Otherwise, we cannot possibly have the guaranteed total $\delta T$ distinguished 
positions in the subsequences on that level, since $(\delta / 2) \cdot 1+(1-\delta / 2) \cdot \delta / 2<\delta$.) Among these, we can count distinct overlap from

$$
\begin{gathered}
\lceil(\delta / 2) 2\rceil=\lceil\delta\rceil \geq \delta / 2-1 / 2 \text { halves, } \\
\lceil(\delta / 2) 4\rceil-\lceil(\delta / 2) 2\rceil=\lceil 2 \delta\rceil-\lceil\delta\rceil \geq \delta-1 / 2 \text { quarters, } \\
\lceil(\delta / 2) 8\rceil-\lceil(\delta / 2) 4\rceil=\lceil 4 \delta\rceil-\lceil 2 \delta\rceil \geq 2 \delta-1 / 2 \text { eighths, } \\
\lceil(\delta / 2) 16\rceil-\lceil(\delta / 2) 8\rceil=\lceil 8 \delta\rceil-\lceil 4 \delta\rceil \geq 4 \delta-1 / 2 \text { sixteenths, } \\
\text { etc. }
\end{gathered}
$$

Unless we find one of these sequences that has relative internal overlap less than $\epsilon$, this accounts, at the $i$ th level, for at least

$$
\left(2^{i-2} \delta-\frac{1}{2}\right)\left(\frac{\epsilon T}{2^{i}}\right)=\frac{\epsilon \delta T}{4}-\frac{\epsilon T}{2^{i+1}}
$$

distinct overlap events, and hence for more than $T$ distinct overlap events after $\lceil(4+2 \epsilon) /(\epsilon \delta)\rceil$ levels. This is impossible, so we must find the desired low-overlap sequence at one of these levels.

2.2. Kolmogorov COMPlexity. A key to the tractability of our arguments (and most of the recent ones we have cited ${ }^{4}$ ) is the use of "incompressible data." Input strings that involve such data tend to be the hardest and least subject to special handling. This general approach is discussed in more detail elsewhere [Paul et al. 1981; Li and Vitányi 1993].

We define incompressibility in terms of Kolmogorov's robust notion of descriptional complexity [Kolmogorov 1965]. Informally, the Kolmogorov complexity $K(x)$ of a binary string $x$ is the length of the shortest binary program (for a fixed reference universal machine) that prints $x$ as its only output and then halts. A string $x$ is incompressible if $K(x)$ is at least $|x|$, the approximate length of a program that simply includes all of $x$ literally. Similarly, a string $x$ is "nearly" incompressible if $K(x)$ is "almost" as large as $|x|$.

The appropriate standard for "almost as large" above can depend on the context, a typical choice being " $K(x) \geq|x|-O(\log |x|)$ ". The latter implicitly involves some constant, however, the careful choice of which might be an additional source of confusion in our many-parameter context. A less typical but more absolute standard such as " $K(x) \geq|x|-\sqrt{|x|}$ " completely avoids the introduction of yet another constant.

Similarly, the conditional Kolmogorov complexity of $x$ with respect to $y$, denoted by $K(x \mid y)$, is the length of the shortest program that, with extra information $y$, prints $x$. And a string $x$ is incompressible or nearly incompressible relative to $y$ if $K(x \mid y)$ is large in the appropriate sense. If, at the opposite extreme, $K(x \mid y)$ is so small that $|x|-K(x \mid y)$ is "almost as large as" $|x|$, then we say that $y$ codes $x$ [Chung et al. 1985].

\footnotetext{
${ }^{4}$ See, for example, Paul [1982; 1984], Paul et al. [1981], Ďriš et al. [1984], Maass [1985], Li and Vitányi [1988], Li et al. [1992], Paturi et al. [1990], and Vitányi [1984].
} 
There are a few well-known facts about these notions that we will use freely, sometimes only implicitly. Proofs and elaboration, when they are not sufficiently obvious, can be found in the literature (especially Li and Vitányi [1993]). The simplest is that, both absolutely and relative to any fixed string $y$, there are incompressible strings of every length, and that most strings are nearly incompressible, by any standard. Another easy one is that significantly long substrings of an incompressible string are themselves nearly incompressible, even relative to the rest of the string. More striking is Kolmogorov and Levin's "symmetry of information" [Zvonkin and Levin 1970]: $K(x)-K(x \mid y)$ is very nearly equal to $K(y)-K(y \mid x)$ (up to an additive term that is logarithmic in the Kolmogorov complexity of the binary encoding of the pair $(x, y))$; that is, $y$ is always approximately as helpful in describing $x$ as vice versa! (Admittedly, the word "helpful" can be misleading here-the result says nothing at all about the relative computational complexity of generating the two strings from each other.) All these facts can be relativized or further relativized; for example, symmetry of information also holds in the presence of help from any fixed string $z$ :

$$
K(x \mid z)-K(x|y| z) \approx K(y \mid z)-K(y|x| z) .
$$

(The meaning of " $K(x|y| z)$ " is the same as that of " $K(x \mid w)$ ", where $w$ is some standard binary encoding of the pair $(y, z)$.)

\section{Proof Strategy}

For the sake of argument, suppose some two-tape Turing machine $M$ does recognize $\left\{x 2 x^{\prime} \mid x \in\{0,1\}^{*}\right.$ and $x^{\prime}$ is a prefix of $\left.x\right\}$ in real time. Once a binary string $x \in\{0,1\}^{*}$ has been read by $M$, the contents of $M$ 's tapes tend to serve as a very redundant representation of prefixes of $x$, because $M$ has had to be prepared to retrieve them at any time. (Our problem and this observation were motivation for Chung, Tarjan, Paul, and Reischuk's investigation of "robust codings of strings by pairs of strings" [Chung et al. 1985]. One way around this would be for $M$ to keep one or the other of its tapes' heads stationed at some stored record of a long prefix of $x$, as "insurance." The early real-time multitape simulations of buffers [Meyer et al. 1967; Fischer et al. 1972; Bennison 1974] do follow this strategy, but we show that a machine with only two tapes will not be able to afford always to use one in this way for insurance: There will have to be a significant subcomputation in which the heads on both tapes "keep moving," even "essentially monotonically" - essentially as they would for straightforward "copying." Under these circumstances, in fact, we will be able to use part of the computation itself, rather than the combination of the two tapes' contents, as the very redundant representation, to contradict the following lemma, which we prove later.

Anti-Holography Lemma. Consider any constant $C$, and consider any binary string $x$ that is long in terms of $C$, and that is nearly incompressible. ${ }^{5}$ Suppose $y=$ $y_{1} y_{2} \ldots y_{k}$ (each $y_{i}$ a binary string) is a "representation" with the following properties:

\footnotetext{
${ }^{5}$ We need $K(x)>\delta|x|$, for some fraction $\delta$ that is determined by $C$; so certainly $K(x)>|x|-\sqrt{|x|}$ will be enough if $x$ is long.
} 
The prefixes of $x$ :

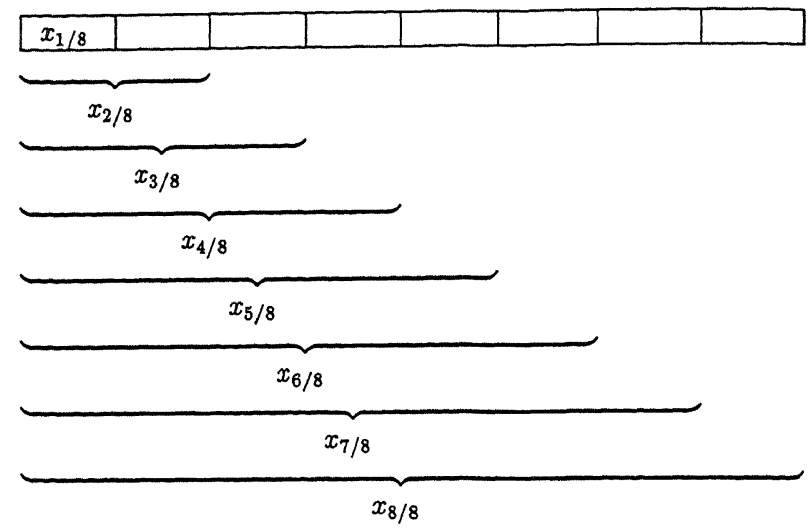

Some of the encoded prefix locations in $y$ :

\begin{tabular}{|c|c|c|c|c|c|c|c|}
\hline$y_{1}$ & $y_{2}$ & $y_{3}$ & $y_{4}$ & $y_{5}$ & $y_{6}$ & $y_{7}$ & $y_{8}$ \\
\hline $\begin{array}{l}x_{1 / 8} \\
\text { here }\end{array}$ & $x_{1 / 8}$ here & $\begin{array}{l}x_{1 / 8} \\
\text { here }\end{array}$ & $\begin{array}{l}x_{1 / 8} \\
\text { here }\end{array}$ & $x_{1 / 8}$ here & $\begin{array}{l}x_{1 / 8} \\
\text { here }\end{array}$ & $\begin{array}{l}x_{1 / 8} \\
\text { here }\end{array}$ & $\begin{array}{l}x_{1 / 8} \\
\text { here }\end{array}$ \\
\hline & here & $x_{2 /}$ & ere & $x_{2 / 8} h$ & & $x_{2 /}$ & here \\
\hline \multicolumn{4}{|c|}{$x_{4 / 8}$ here } & \multicolumn{4}{|c|}{$x_{4 / 8}$ here } \\
\hline
\end{tabular}

FIG. 1. "Holography" example $(k=8)$.

(1) $|y| \leq C|x|$;

(2) For each $\ell \leq k$, $x$ 's prefix " $x_{\ell / k}$ " of length $\ell|x| / k$ is coded by $y_{i+1} \cdots y_{i+\ell}$ for each $i \leq k-\ell$.

(See Figure 1.) The $k$ is bounded by some constant that depends only on $C$.

For (the binary representation of) a $T$-step subcomputation by $M$ to serve as a representation $y$ that contradicts this lemma, we need the following:

(1) A nearly incompressible input prefix $x$ of length at least $|y| / C=\Theta(T / C)$ was read before the subcomputation.

(2) There is a parse of the subcomputation into a large number $k$ of pieces so that each $x_{\ell / k}$ is coded in every contiguous sequence of $\ell$ pieces.

(3) $k$ is (too) large in terms of $C$.

We accomplish these things by finding a subcomputation that has a spatially monotonic "matching" (a notion intended to model aspects of standard "copying") that is both long and so well separated spatially that needed information on tape contents cannot be spread over many pieces of the subcomputation.

The first step is to define and find "a large matching," and the second is to refine it in a suitable way. In a two-tape or two-head computation or subcomputation, a monotonic sequence of time instants is a matching if neither head scans the same tape square at more than one of the time instants. (So there is actually a separate one-to-one "matching" for each head, between the time instants and 


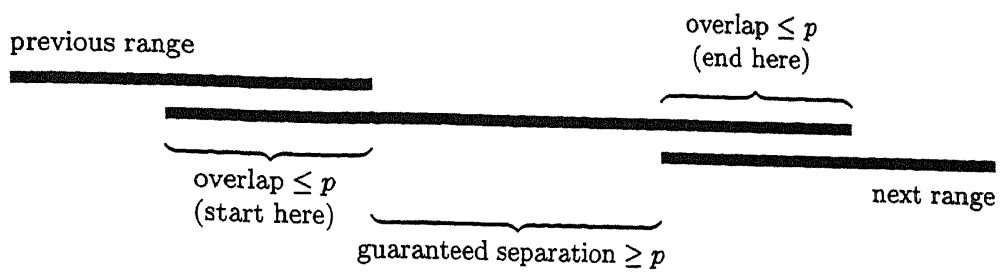

FIG. 2. Range of a rightward head.

the tape squares scanned by that head at those times.) We prove the following lemma later on.

LARGE-MATCHING LEMMA. If a two-tape Turing machine recognizes

$$
\left\{x 2 x^{\prime} \mid x \in\{0,1\}^{*} \text { and } x^{\prime} \text { is a prefix of } x\right\}
$$

in real time, then its computation on an incompressible binary input of length $n$ includes a matching of length $\Omega(n)$. (The implicit constant does depend on the machine.)

(Note that this lemma does not hold if the two heads can be on the same tape, since the straightforward real-time recognizer leaves one head completely stationary until the symbol 2 is reached.)

In a two-tape or two-head computation or subcomputation, a matching is (spatially) monotonic if, for each of the two heads, the spatial order of the corresponding sequence of tape squares being scanned at the specified time instants is strictly left-to-right or strictly right-to-left. The minimum separation of a monotonic matching is the least distance between successive tape squares ir either corresponding sequence of tape squares.

Monotonization LEMMA. If a multitape or multihead computation or subcomputation has a matching of length $m$ and internal overlap at most $p$, then it has a monotonic submatching of length at least $\lceil m\lceil 3 p\rceil\rceil$ and minimum separation at least $p$.

Proof. Just choose every $\lceil 3 p\rceil$-th matching-time instant, starting with the first.

To see that this works, consider parsing the computation into $\lceil m /\lceil 3 p\rceil\rceil$ subcomputations, each (except possibly the last) including a matching of length at least $3 p$. Because the tapes are one-dimensional, each subcomputation involves a contiguous set, or "range", of at least $3 p$ distinct tape squares visited by each head. Because of the overlap bound, these ranges overlap by no more than $p$, and each head begins and ends each subcomputation within $p$ of opposite ends of its range for that subcomputation. (See Figure 2.)

\section{Careful Argument}

Now let us put together the whole argument, taking care to introduce the various "constants" in an appropriate order $-M$ (and $d$ ), then $\delta$, then $\epsilon$, and finally $\epsilon^{\prime}$-all before the input length $n$ and the particular input string $x_{0}$ on which we focus. Each of these values is allowed to depend on earlier ones, but not on later ones. 
For the sake of argument, suppose some two-tape Turing machine $M$ does recognize the language $\left\{x 2 x^{\prime} \mid x \in\{0,1\}^{*}\right.$ and $x^{\prime}$ is a prefix of $\left.x\right\}$ in real time, say with delay bound $d$. Citing the Large-Matching Lemma, take $\delta>0$ small enough so that $M$ 's computation on any sufficiently long incompressible input string $x \in\{0,1\}^{*}$ includes a matching of length at least $2 \delta|x|+2$. Let $\epsilon>0$ be small in terms of $d, \delta$, and $M$; and let $\epsilon^{\prime}$ be small in terms of $d, \delta$, and $\epsilon$. Let $n$ be large in terms of all these constants, and let $x_{0}$ be any incompressible string of $n$ bits.

Split the computation by $M$ on input $x_{0}$ into an initial subcomputation and a final subcomputation, each including a matching of length at least $\delta n$. The number of steps in each of these subcomputations will lie between $\delta n$ and $d n$. Therefore, the initial one will involve a prefix of $x_{0}$ of length at least $\delta n / d$, and the final one will have "match density" at least $(\delta n) /(d n)=\delta / d$.

Applying the Overlap Lemma to the final subcomputation above, we obtain a subcomputation of some length $T \geq \epsilon^{\prime} n$, with match density at least $\delta /(4 d)$ and relative internal overlap less than $\epsilon$, provided $\epsilon^{\prime}$ was chosen small enough in terms of $d, \delta$, and $\epsilon$. Then applying the Monotonization Lemma, we obtain within this subcomputation a monotonic submatching of minimum separation at least $\epsilon T$, and of length $2 k+1$, where $2 k+1$ is either $\lceil(\delta /(4 d)) T /\lceil 3 \epsilon T\rceil\rceil$ or $\lceil(\delta /(4 d)) T /\lceil 3 \epsilon T\rceil\rceil-1$ (whichever is odd). If $\epsilon$ was chosen small, then $k$ will be large. Note that $k \epsilon$ is approximately equal to a constant $\delta /(24 d)$ that depends only on $M$.

To obtain the desired contradiction to the Anti-Holography Lemma, take $y$ to be a complete record of the $T$-step subcomputation obtained above, including the symbols scanned and written by each head on each step. To obtain $y_{1}$, $y_{2}, \ldots, y_{k}$, split this record at every second one of the time instants corresponding to the matching of length $2 k+1$, starting with the third and ending with the third-to-last. Take $x$ to be $x_{0}$ 's prefix of length $\lfloor k \in T / d\rfloor$. Since $\delta n / d$ exceeds this length (by a factor of at least about 24 , since $T \leq d n$ ), all of $x$ was already read during the initial subcomputation above, and hence before the beginning of the subcomputation described by $y$. Note that, for some constant $D$ that depends only on $M$,

$$
|y| \leq D T \approx \frac{d D}{k \epsilon}|x| \approx \frac{24 d^{2} D}{\delta}|x|,
$$

and that $k$ is large (in fact, too large for the Anti-Holography Lemma) in terms of the constant $C=24 d^{2} D / \delta$, assuming we chose $\epsilon$ small enough.

To see that $x_{\ell / k}$ is coded by $y_{i+1} \cdots y_{i+\ell}$ (for each appropriate $\ell$ and $i$ ), suppose we interrupt $M$ with "the command to begin retrieval" (i.e., with the symbol 2) at the $(2 i+\ell+1)$-st of the time instants corresponding to the matching of length $2 k+1$. (This is the middle match point in $y_{i+1} \cdots y_{i+\ell}$.) Since $M$ must be able to check $x_{\ell / k}$ by reading only the information within distance $d \ell|x| / k \leq \ell \epsilon T$ of its heads, that prefix must be coded by that information. Since this distance in each direction is just $\ell$ times the minimum separation of the matching, and since the matching is monotonic, the same information is available within the subcomputation record $y$, between the matching's time instants $2 i+\ell+1-\ell$ and $2 i+\ell+1+\ell$. Since 
$y_{i+1} \cdots y_{i+\ell}$ runs from the matching's time instant $2 i+1$ to the matching's time instant $2 i+2 \ell+1$, therefore, it does code the desired prefix.

\section{Proof of Anti-Holography Lemma}

Recall from above the statement of the lemma (and Figure 1). Without loss of generality, assume $k$ is equal to $2^{e}$ for some integer exponent $e .^{6}$ Then the target constant can be $2^{2 C-1}$. Again without loss of generality, assume $k$ is at most this target constant times two. ${ }^{7}$ Finally, without loss of generality, assume that $|x|=n$ is divisible by $k$, with $x=x_{1} \ldots x_{k}$ and $\left|x_{i}\right|=n / k$ for every $i .^{8}$

The key idea is to obtain dramatically shortened descriptions of $y$ in terms of prefixes of $x$. Since each prefix of $x$ is coded in many disjoint substrings of $y$, we can use symmetry of information to efficiently abbreviate all those substrings in terms of the same one prefix of $x$. For each $j \leq e$, and for $j=e-1$ in particular, this approach will yield

$$
K\left(y \mid x_{1} \cdots x_{2 j}\right) \leq|y|-\left(1+\frac{j}{2}\right) n+\mathcal{O}(\log n) .
$$

Unless $k$ is smaller than $2^{2 C-1}, e-1$ will be so large that this will imply that $x_{1} \cdots x_{2^{e-1}}$ codes $y$. Since $y$ in turn codes all of $x=x_{1} \cdots x_{2^{e}}$, this will mean that the first half of $x$ codes the whole string, contradicting the incompressibility assumption for $x$.

By induction on $j(j=0,1, \ldots, e)$, we actually prove "more local" bounds that imply the ones above: For each appropriate $i\left(i=0,1, \ldots, k-2^{j}\right)$,

$$
K\left(y_{i+1} \cdots y_{i+2 j} \mid x_{1} \cdots x_{2 j}\right) \leq\left|y_{i+1} \cdots y_{i+2 j}\right|-2^{j}\left(1+\frac{j}{2}\right) \frac{n}{k}+\mathcal{O}(\log n) .
$$

Both the base case and the induction step are applications of an intuitively clear corollary, the Further-Abbreviation Lemma below, of the symmetry of information. For the base case, we apply the lemma with $y^{\prime}$ equal to $y_{i+1}, x^{\prime}$ equal to the null string, and $x^{\prime \prime}$ equal to $x_{1}$, to get the desired bound on $K\left(y^{\prime} \mid x^{\prime \prime}\right)$ :

$$
\begin{aligned}
K\left(y^{\prime} \mid x^{\prime \prime}\right) & \leq K\left(y^{\prime}\right)-K\left(x^{\prime \prime}\right)+\mathcal{O}(\log n) \\
& \leq\left|y^{\prime}\right|-\frac{n}{k}+\mathcal{O}(\log n) .
\end{aligned}
$$

For the induction step, we let $y^{\prime \prime}=y_{i+1} \cdots y_{i+2^{j}}$ and $y^{\prime \prime \prime}=y_{i+2^{j}+1} \cdots y_{i+2^{j+1}}$, and apply the lemma with $y^{\prime}$ equal to $y^{\prime \prime} y^{\prime \prime \prime}, x^{\prime}$ equal to $x_{1} \ldots x_{2^{j}}$, and $x^{\prime \prime}$ equal to

\footnotetext{
${ }^{6}$ If it is not, then just reduce it until it is.

7 Otherwise, pair up $y_{i}$ 's to reduce $k$ by factors of 2 until it is.

${ }^{8}$ If $x$ 's length is not divisible by $k$, then just discard at most its last $2^{2 C-1}$ bits, until its length is divisible by $k$.
} 
$x_{2^{j}+1} \cdots x_{2}^{j+1}$, to get the desired bound on $K\left(y^{\prime} \mid x^{\prime} x^{\prime \prime}\right)$ :

$$
\begin{aligned}
K\left(y^{\prime} \mid x^{\prime} x^{\prime \prime}\right) & \leq K\left(y^{\prime} \mid x^{\prime}\right)-K\left(x^{\prime \prime}\right)+\mathcal{O}(\log n) \\
& \leq K\left(y^{\prime \prime} \mid x^{\prime}\right)+K\left(y^{\prime \prime \prime} \mid x^{\prime}\right)-K\left(x^{\prime \prime}\right)+\mathcal{O}(\log n) \\
& \leq\left|y^{\prime \prime}\right|+\left|y^{\prime \prime \prime}\right|-2 \cdot 2^{j}\left(1+\frac{j}{2}\right) \frac{n}{k}-2^{j} \frac{n}{k}+\mathcal{O}(\log n) \\
& =\left|y^{\prime \prime}\right|+\left|y^{\prime \prime \prime}\right|-2^{j+1}\left(1+\frac{(j+1)}{2}\right) \frac{n}{k}+\mathcal{O}(\log n) .
\end{aligned}
$$

Further-Abbreviation Lemma. Assume $y^{\prime}, x^{\prime}$, and $x^{\prime \prime}$ are strings of length $\Theta(n)$, with

$$
K\left(x^{\prime \prime} \mid y^{\prime}\right)=\mathcal{O}(\log n)
$$

and

$$
K\left(x^{\prime \prime} \mid x^{\prime}\right)=K\left(x^{\prime \prime}\right)-\mathcal{O}(\log n) .
$$

(That is, $y^{\prime}$ codes $x^{\prime \prime}$, which is nearly incompressible relative to $x^{\prime}$.) Then

$$
K\left(y^{\prime} \mid x^{\prime} x^{\prime \prime}\right) \leq K\left(y^{\prime} \mid x^{\prime}\right)-K\left(x^{\prime \prime}\right)+\mathcal{O}(\log n) .
$$

Proof. Let $d(u \mid v)$ denote a shortest description of $u$ in terms of $v$, so that $|d(u \mid v)|=K(u \mid v)$. Then

$$
\begin{aligned}
K\left(y^{\prime} \mid x^{\prime} x^{\prime \prime}\right) & \leq K\left(d\left(y^{\prime} \mid x^{\prime}\right) \mid x^{\prime} x^{\prime \prime}\right)+\mathcal{O}(\log n) \\
& \leq K\left(d\left(y^{\prime} \mid x^{\prime}\right)\left|x^{\prime \prime}\right| x^{\prime}\right)+\mathcal{O}(\log n) \\
& \leq K\left(d\left(y^{\prime} \mid x^{\prime}\right) \mid x^{\prime}\right)-K\left(x^{\prime \prime} \mid x^{\prime}\right)+K\left(x^{\prime \prime}\left|d\left(y^{\prime} \mid x^{\prime}\right)\right| x^{\prime}\right)+\mathcal{O}(\log n) \\
& \leq K\left(y^{\prime} \mid x^{\prime}\right)-K\left(x^{\prime \prime} \mid x^{\prime}\right)+K\left(x^{\prime \prime} \mid y^{\prime}\right)+\mathcal{O}(\log n) \\
& \leq K\left(y^{\prime} \mid x^{\prime}\right)-K\left(x^{\prime \prime}\right)+\mathcal{O}(\log n) .
\end{aligned}
$$

\section{Proof of Large-Matching Lemma}

Our proof of the Large-Matching Lemma is based on an earlier theorem of Vitányi:

FAR-OUt LEMMA. [VITÁNYI 1984]. ${ }^{9}$ If a two-tape Turing machine recognizes $\left\{x 2 x^{\prime} \mid x \in\{0,1\}^{*}\right.$ and $x^{\prime}$ is a prefix of $\left.x\right\}$

${ }^{9}$ For a sketch of the proof, see the appendix. 


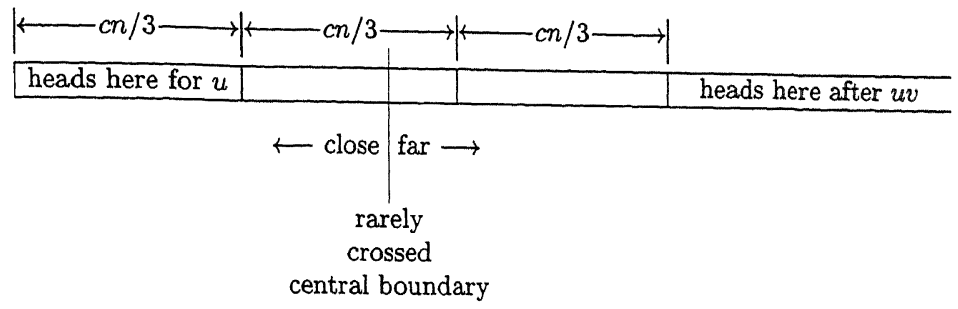

FIG. 3. M's computation on $u v$.

in real time, then its "worst-case closest head position" 10 on incompressible inputs $x \in\{0,1\}^{n}$ is $\Omega(n)$.

In other words, incompressible binary data is guaranteed at some point to drive both heads of such a machine simultaneously far from their original positions. By the continuity of sequential access, of course, this means that the heads actually spend long intervals of time simultaneously far from their original positions; and this is the fact that we exploit.

We actually show that even any two-head Turing machine (with both heads on the same one-dimensional tape) that recognizes our language and that satisfies the conclusion of the Far-Out Lemma also satisfies the desired conclusion of the Large-Matching Lemma. (Of course, the obvious two-head machine, that does recognize our language in real time, does not satisfy the conclusion of either lemma.) This simplifies the exposition, since we have only one tape to talk about. Note that the "matching" notion does make sense even when both heads are on the same tape.

As earlier, let us take explicit care to introduce our "constants" in an appropriate order. Consider any two-head Turing machine $M$ alleged to recognize

$$
\left\{x 2 x^{\prime} \mid x \in\{0,1\}^{*} \text { and } x^{\prime} \text { is a prefix of } x\right\}
$$

in real time, say with delay bound $d$, and that satisfies the conclusion of the Far-Out Lemma. (Without loss of generality, assume $M$ 's tape has a left end, where both heads start, and is unbounded to the right only.) Let $c$ be small enough to serve as the implicit constant in that conclusion. Let $\epsilon$ be small in terms of $M$ and $c$; let $\delta$ be small in terms of $M, c$, and $\epsilon$; let $n$ be large in terms of $M, c, \epsilon$, and $\delta$; and let $x$ be an incompressible string of $n$ bits. Exploiting the conclusion of the Far-Out Lemma, parse $x$ into three pieces, $x=u v w$, such that $u v$ leaves both heads at least $c n$ tape squares from where they started and the length of $u$ is $\lfloor c n /(3 d)\rfloor=\Theta(n)$.

Consider $M$ 's computation on $u v 2 u$. The first $u$ must be read before either head gets as far as even $c n / 3$ tape squares from where it started, but the second $u$ must be read while neither head gets closer than $2 c n / 3$ tape squares to where it started. (See Figure 3.) During its subcomputation on $v$, therefore, it seems that $M$ must somehow "copy" its representation of $u$ across the intervening $c n / 3$ tape squares. We show that this process has to involve a matching larger than $\delta n$.

${ }^{10}$ If $p_{i}(t)$ denotes the net displacement of head $i$ at time $t$, then the "worst-case closest head position" is $\max _{t} \min _{i} p_{i}(t)$. 
For the sake of argument, suppose there is not a matching larger than $\delta n$. Then there must be a maximal matching of size only $m \leq \delta n$. We will select some correspondingly small "interface" through which a description of $u$ must pass. That interface will involve some rarely crossed boundary at distance between $c n / 3$ and $2 c n / 3$ from the heads' starting position, and some other rarely crossed boundaries that tightly isolate the $2 m$ tape squares involved in the maximal matching. (Think of the latter tape squares as "shortcut terminals" through which we can "ship" information about $u$ without a head carrying it. Maximality will ensure that there is always at least one head on one of these terminals.) Since there are $2 c n / 3-c n / 3$ candidates for the former, we can select one that is crossed only a constant number of times (bounded in terms of $d$ and $c$ ). We will refer to the tape squares on the left side of this selected central boundary, where the heads were when $u$ was read, as close; and we will refer to the tape squares on the right side as far. (See Figure 3 again.) By the following purely combinatorial lemma, we can tightly isolate the $2 m$ matched tape squares with at most $4 m$ additional boundaries, each of which is crossed only a constant number of times (bounded in terms of $d, c$, and our "tightness criterion" $\epsilon$ ).

Tight-Isolation LEMma. Consider a finite sequence $S$ of nonnegative numbers, the first and last of which are 0. Let some of the separating "commas" be specially designated-call them "semicolons". For each threshold $\ell \geq 0$, let $S_{\ell}$ be the subsequence (not necessarily contiguous) consisting of the items ${ }^{11}$ that are reachable from the semicolons via items that exceed $\ell$ (and that themselves exceed $\ell$ ). Then, for each $\epsilon>0$, there is some $\ell$ such that $\ell\left|S_{\ell}\right|<\epsilon \Sigma S$, where $\sum S$ denotes the sum of the entire sequence $S$ and $\ell$ is bounded by some constant that depends only on $\epsilon$.

Proof. Let $T=\sum S$, and let $k=2\lceil 2 / \epsilon\rceil$. Since $2 T / k \leq \epsilon T / 2<\epsilon T$, let us aim for $\ell\left|S_{\ell}\right| \leq 2 T / k$. If no $\ell$ in $\left\{k^{i} \mid 0 \leq i \leq k\right\}$ were to work, then we would have

$$
2 \frac{T}{k}<k^{i}\left|S_{k^{i}}\right|<T
$$

for every $i$. But this would lead to the contradiction

$$
\begin{aligned}
T & >\sum_{i=0}^{k} k^{i}\left(\left|S_{k^{i}}\right|-\left|S_{k^{i+1}}\right|\right) \\
& >\sum_{i=0}^{k}\left(\frac{2 T}{k}-\frac{T}{k}\right) \\
& =(k+1) \frac{T}{k} .
\end{aligned}
$$

\footnotetext{
${ }^{11}$ Note that the number of such items can be small even if the number of semicolons is large. For $\ell$ large enough, in fact, $\left|S_{\ell}\right|$ will be 0 .
} 
In our application, the numbers are the lengths of the crossing sequences associated with the boundaries between tape squares, their sum is at most $d n$, and the semicolons are the matched tape squares. We obtain our desired "isolation neighborhoods" from the at-most $-2 m$ contiguous neighborhoods that comprise $S_{\ell}{ }^{12}$ by adding one item at each end of each neighborhood. (This might cause neighborhoods to combine.) This adds at most $4 m$ items to $S_{\ell}$ and results in nonempty isolation neighborhoods whose boundary items are at most $\ell$.

Actually, the picture is clearer if we select our central boundary after we select the isolation neighborhoods. Assuming $\epsilon$ and $\delta$ are chosen appropriately small, this lets us select a boundary not included in or adjacent to any of the isolation neighborhoods. (There are at most $\left|S_{\ell}\right|+4 m \leq \epsilon d n+4 \delta n \leq c h / 6$ boundaries (half the original number of candidates) to avoid.)

Finally, we use our suggested interface to give a description of $u$ in terms of $v$ that is too short-say shorter than $|u| / 2 \approx c n /(6 d)$. (We could substitute a description this short for $u$ in $x$ to contradict the incompressibility of $x$.) We claim we can reconstruct $u$ from $M, v$, the length of $u$, and the following information about the subcomputation of $M$ while reading the $v$ part of input $u v$ :

(1) The sequence of all $O(m)$ selected boundary locations.

(2) The sequence of all $O(m)$ crossings of these selected boundaries, and their times (implicitly or explicitly including the corresponding input positions).

(3) The following information for each close-to-far crossing:

$-M$ 's control state and head positions.

- The full content of every isolation neighborhood.

(4) The following information for each crossing out of an isolation neighborhood:

- The full content of that isolation neighborhood.

- The full content of the isolation neighborhood in which the other head remains-provided that there has been a new crossing into that neighborhood since the previous time such information was given for it.

To determine $u$, it suffices to reconstruct enough of $M$ 's configuration after its computation on input $u v$ so that we can check which additional input string $2 u^{\prime}$ of length $1+|u|$ leads to acceptance. The far tape contents suffice for this.

Our reconstruction strategy is mostly to simulate $M$ step by step, starting with the first close-to-far crossing. During step-by-step simulation, we maintain the contents of any currently scanned close isolation neighborhood and of the entire far side. We temporarily suspend step-by-step simulation whenever a head shifts onto a close tape square not in any isolation neighborhood (at which time the other head must lie within some isolation neighborhood). We aim to resume suspended step-by-step simulation whenever a head shifts onto a far tape square not in any isolation neighborhood. Between a suspension and the desired resumption, the far tape contents are not scanned and do not change, except in the (at most) one scanned isolation neighborhood. Therefore, since the desired resumption is inaugurated by either a close-to-far crossing or a crossing out of

\footnotetext{
${ }^{12}$ To include all the semicolons, some of these "contiguous neighborhoods" might have to be the empty neighborhoods of the semicolons.
} 
that one isolation neighborhood, it follows that all the prerequisite information for resumption is indeed available at resumption time. Finally, since both heads are on the far side when the computation on $u v$ ends, our step-by-step simulation is active at that point, and we do obtain the desired far tape contents.

It remains only to show that $|u| / 2$ bits suffice for our description of $u$ in terms of $v$. For each of the sequences in (1) and (2), the trick is to give only the first number explicitly, and then to give the sequence of successive differences. The length of this encoding is $O(m \log (n / m))=\mathcal{O}(n \log (1 / \delta) /(1 / \delta))$, which can be limited to a small fraction of $|u| / 2 \approx c n /(6 d)$ by choosing $\delta$ small enough. For (4), note that that the contents of each isolation neighborhood is given at most once for each of the (at most) $\ell$ crossings into and out of the neighborhood. For (3) and (4), therefore, straightforward encoding requires only $\mathcal{O}(\log n+\ell(m+$ $\left.\left.\left|S_{\ell}\right|\right)\right)=\mathcal{O}(\log n+\ell \delta n+\epsilon d n)$ bits, where the implicit constant is bounded in terms of $d$ and $c$. This can be limited to another small fraction of $|u| / 2$ by choosing $\epsilon$ small enough, $\delta$ small enough, and $n$ large enough. For the remaining information, $M,|u|$, and a description of this whole discussion, we need only $O(\log n)$ bits, which can be limited to a final small fraction of $|u| / 2$ by choosing $n$ large enough.

\section{Further Discussion and Remaining Questions}

In retrospect, our contribution has been a constraint on how a Turing machine with only two storage heads can recognize $L$ in real time. Even if the two heads are on the same one-dimensional tape, such a Turing machine cannot recognize $L$ in real time unless it violates the conclusion of (the first sublemma of) Vitányi's Far-Out Lemma (see Appendix A). Only in the latter do we ever really exploit an assumption that the two heads are on separate tapes.

Our result rules out general real-time simulation of a two-head tape unit using only a pair of single-head tapes. It remains to be investigated whether the result extends to some notion of probabilistic real-time simulation (cf., Paturi et al. [1990]). Another extension might rule out simulation using three single-head tapes, yielding a tight result; but this would require a more difficult witness language. Perhaps allowing the "back" head of the defining two-head machine also to move and store random data, but much more slowly than the "front" head, would let us combine our arguments with those of Aanderaa [Aanderaa 1974; Paul et al. 1981; Paul 1982]. A slightly weaker possibility might be to show that two single-head tapes and a pushdown store do not suffice, and a slightly stronger one might be to show that even three single-head tapes and a pushdown store do not suffice.

It might be even more difficult to rule out general real-time simulation of a two-head one-dimensional tape unit using two or three higher-dimensional single-head tapes. Our particular language $L$ can be recognized in real time by a Turing machine with just two such two-dimensional tapes-the idea is to strive to maintain the $n$ bits of data within an $O(\sqrt{n})$ radius on both tapes, along with $O(\sqrt{n})$ strategically placed copies of the first $O(\sqrt{n})$ bits, to serve as insurance alternatives at the same time that the array of their left ends provides a convenient area for temporary collection of data and for copying data between the tapes. 
The implications for real-time simulation of one-dimensional tape units with more than two heads remain to be investigated. For example, how does a three-head tape compare with three single-head tapes or with one single-head tape and one two-head tape? (Paul's results [Paul 1984] do answer such questions for tapes of higher dimension.) How tight is the known bound of $4 h-$ 4 single-head tapes for real-time simulation of one $h$-head (one-dimensional) tape [Leong and Seiferas 1981]? Perhaps the many-heads setting is the right one for a first proof that even an extra head is not enough to compensate for the loss of sharing; for example, can a 1000-head tape be simulated in real time by 1001 single-head tapes, or by 1000 single-head tapes and a pushdown store?

Finally, does any of this lead to more general insight into the heads or tapes requirements for arbitrary computational tasks? That is, when asked about some computational task, can we tightly estimate the structure of the sequential storage that suffices for the task?

\section{Appendix A. A Proof Sketch for Vitányi's Far-Out Lemma}

Suppose two-tape Turing machine $M$ recognizes the language in real time. Without loss of generality, assume $M$ 's storage tape is only semi-infinite, and assume $M$ writes only 0 's and 1's. Let $d$ be the delay of $M$.

Our ultimate goal is to show that both heads simultaneously range linearly far when the input is incompressible, but first we show that each one separately does so even when the input is just nearly incompressible. (The subsequent application is to significantly long prefixes of input strings that are not compressible at all.) It is only this part of the proof that requires the hypothesis that the two heads are on separate tapes. Like the proof of our Large-Matching Lemma above, this part is based on the "bottleneck" argument that Valiev [1970/1973] (and, independently, Meyer [1971]) used to show that no single-tape Turing machine can accept the simpler language $\left\{x 2 x \mid x \in\{0,1\}^{*}\right\}$ in real time.

Suppose $\epsilon$ is small in terms of $M$ and $d, n$ is large in terms of all of the preceding, and $x$ is of length $n$ and nearly incompressible $(K(x) \geq n-\sqrt{n})$. We want to show that each head ranges farther than $\epsilon n$.

Suppose the head on one of the tapes, say the first, does not range farther than $\epsilon n$. Then the head on the second tape must certainly range farther than, say, $n / 3$. (Otherwise, the total state after storage of $x$ is a too-short description of $x$.) Let $u v w$ be the parse of $x$ with $u v$ the shortest prefix of $x$ that leaves $M$ 's second head at least $n / 3$ tape squares out, and with $|u|=n /(9 d)$, so that that same head gets no farther than $n / 9$ tape squares out during input of $u$. On that head's tape, there must be a "bottleneck" boundary between $n / 9$ and $2 n / 9$ tape squares out that gets crossed at most $9 d$ times (cf. Figure 3). Since all of $u$ gets read when the second head is to the left of this bottleneck, it is possible to describe $x=$ $u v w$ in terms of $v w$ and the bottleneck's "crossing sequence," which should include, for each crossing, the step number and the "crossing state," which in turn should include the complete but relatively small contents of the first storage tape at the time of the crossing. The following information suffices:

(1) $v w$,

(2) a description of this discussion,

(3) a description of $M$, 
(4) the value of $n$,

(5) the location of the bottleneck,

(6) the crossing sequence at the bottleneck.

If we provide $v w$ as a literal suffix, then we can limit the length of this description to little more than $n-|u|$ bits, contradicting the near incompressibility of $x$. To recover $u$, we can use the information to determine enough of $M$ 's instantaneous description after reading $u v$ (omitting from the instantaneous description only what is to the left of the bottleneck on the second tape) to then try each input continuation $2 u^{\prime}$ with $\left|u^{\prime}\right|=n /(9 d)$.

Finally, we return to our ultimate goal. Here is the idea: If the heads do not both go far out together, then they must take turns, so that some region gets crossed many times; abbreviate the symbols read while a head is in that region.

Suppose $\epsilon$ is small in terms of $M$ and $d$ (as above), $\epsilon_{2}$ is small in terms of the preceding parameters (in particular, $\epsilon_{2} \ll \epsilon$ ), $\epsilon_{1}$ is small in terms of the now preceding parameters (in particular, $\epsilon_{1} \ll \epsilon_{2}$ ), $n$ is large in terms of all of the above, and $x$ is of length $n$ and incompressible. We want to show that both heads range farther than $\epsilon_{1} n$, simultaneously.

Suppose, to the contrary, that there is always at least one head within $\epsilon_{1} n$ tape squares of the origin. We count the crossings of the region from $\epsilon_{1} n$ to $\epsilon_{2} n$ : It follows from our assumptions that a left-to-right crossing must occur between input symbol number $(d / \epsilon)^{i}\left(\epsilon_{2} n / \epsilon\right)$ and input symbol number $(d / \epsilon)^{i+1}\left(\epsilon_{2} n / \epsilon\right)$, for every $i$. (We use the fact that these input prefixes are themselves nearly incompressible.) By input symbol number $n$, therefore, the number of complete crossings (either direction) is at least $r=2 \log _{d / \epsilon}\left(\epsilon / \epsilon_{2}\right)$ (which is large because $\epsilon_{2}$ is so small).

There is a complication, however: There might also be partial crossings, involving fewer input symbols but additional overhead in the description we plan to give. To control this problem, we shrink the region slightly, replacing $\epsilon_{1}$ and $\epsilon_{2}$ with $\epsilon_{1}^{\prime}$ and $\epsilon_{2}^{\prime}$ from the first and last quarters, respectively, of the range $\left[\epsilon_{1}, \epsilon_{2}\right]$, chosen so that each of the boundaries $\epsilon_{1}^{\prime} n$ and $\epsilon_{2}^{\prime} n$ is crossed at most $R=8 d / \epsilon_{2}$ times. This is possible, since $R\left(\epsilon_{2}-\epsilon_{1}\right) n / 4$ exceeds $d n$.

Finally, then, we formulate a description of the incompressible input that differs from the completely literal one as follows: We eliminate the input read while a head is in the range between $\epsilon_{1}^{\prime} n$ and $\epsilon_{2}^{\prime} n$, for a savings of at least $r\left(\epsilon_{2}^{\prime}-\right.$ $\left.\epsilon_{1}^{\prime}\right) n / d \geq r\left(\epsilon_{2}-\epsilon_{1}\right) n /(2 d)$ bits. We add descriptions of the crossing sequences at these two boundaries, including times, states, and the tape contents out to boundary $\epsilon_{1} n$ (the second term below), and also the full final contents of the tape squares between the two boundaries (the first term below), for a total cost of

$$
\begin{aligned}
\mathcal{O}\left(\left(\epsilon_{2}^{\prime}-\epsilon_{1}^{\prime}\right) n+R\left(\log n+\epsilon_{1} n\right)\right) & =\mathcal{O}\left(\left(\epsilon_{2}-\epsilon_{1}\right) n+\frac{8 d\left(\log n+\epsilon_{1} n\right)}{\epsilon_{2}}\right) \\
& =\mathcal{O}\left(\left(\epsilon_{2}-\epsilon_{1}\right) n\right)
\end{aligned}
$$

bits, which can be kept significantly smaller than the savings.

ACKNOWLEDGMENTS. The authors thank Wolfgang Maass for discussions that contributed to an early version of the Anti-Holography Lemma in 1985. The authors also thank Ming $\mathrm{Li}$ for other valuable discussions, and for first bringing 
the problem addressed here to the attention of $\mathrm{T}$. Jiang. Thanks are also due to Zvi Galil, Ken Regan, and the anonymous referee for helpful comments on the manuscript.

\section{REFERENCES}

AANDERAA, S. O. 1974. 1974. On $k$-tape versus $(k-1)$-tape real time computation. In Complexity of Computation (SLAM-AMS Proceedings 7) R. M. Karp. ed. American Mathematical Society, Providence, R. I., pp. 75-96.

BEČVÁř, J. 1965. Real-time and complexity problems in automata theory. Kyberntika 1, 6, 475497.

BenNison, V. L. 1974. Saving tapes in the simulation of multihead Turing machines. SIGACT News 6, 2 (Apr.), 23-26.

Chung, F. R. K., TARJAN, R. E., Paul, W. J., AND ReIschuK, R. 1985 . Coding strings by pairs of strings. SIAM J. Disc. Math. 6, 3 (July), 445-461.

Ďuriš, P., Galil, Z., Paul, W. J., AND Reischuk, R. 1984. Two nonlinear lower bounds for on-line computations. Inf. Cont. 60, 1-3 (Jan.-Mar.), 1-11.

Fischer, P. C., Meyer, A. R., ANd Rosenberg, A. L. 1972. Real-time simulation of multihead tape units. J. $A C M 19,4$ (Oct.), 590-607.

GRIGORIEV, D. YU. 1977. Imbedding theorems for Turing machines of different dimensions and Kolmogorov's algorithms. Sov. Math. 18, 3 (May-June), 588-592.

HaRTMAnis, J., AND STEaRnS, R. E. 1965. On the computational complexity of algorithms. Trans. Am. Math. Soc. 117, 5 (May), 285-306.

HeNNIE, F. C. 1966. On-line Turing machine computations. IEEE Trans. Elect. Comput. EC-15, 1 (Feb.), 35-44.

Kolmogorov, A. N. 1965. Three approaches to the quantitative definition of information. Prob. Inf. Transm. 1, 1 (Jan.-Mar.), 1-7.

LEONG, B. L., AND SEIfERAS, J. I. 1981. New real-time simulations of multihead tape units. J. $A C M$ 28,1 (Jan.), 166-180.

Li, M., Longré, L., ANd Vitányi, P. M. B. 1992. The power of the queue. SIAM J. Comput. 21, 4 (Aug.), 697-712.

LI, M., AND VitÁNyi, P. M. B. 1988. Tape versus queue and stacks: The lower bounds. Inf. Comput. 78, 1 (July), 56-85.

LI, M., And Vitányi, P. M. B. 1993. An Introduction to Kolmogorov Complexity and Its Applications. Springer-Verlag, New York.

MAASS, W. 1985. Combinatorial lower bound arguments for deterministic and nondeterministic Turing machines. Trans. Am. Math. Soc. 292, 2 (Dec.), 675-693.

MaAss, W., Schnitger, G., Szemerédi, E., And Turán, G. 1993. Two tapes versus one for off-line Turing machines. Comput. Complex. 3, 4, 392-401.

MEYER, A. R. 1971. An optimal time bound for a one tape on-line Turing machine computation. Unpublished manuscript. (Earlier version cited in MEYER ET AL. 1967.)

Meyer, A. R., Rosenberg, A. L., AND Fischer, P. C. 1967. Turing machines with several read-write heads, preliminary report. In IEEE Conference Record of 1967 8th Annual Symposium on Switching and Automata Theory. IEEE Computer Society, Long Beach, Calif., pp. 117-127.

Paturi, R., Seiferas, J. I., Simon, J., And Newman-Wolfe, R. E. 1990. Milking the Aanderaa argument. Inf. Comput. 88, 1 (Sept.), 88-104.

Paul, W. J. 1982. On-line simulation of $k+1$ tapes by $k$ tapes requires nonlinear time. Inf. Cont. 53, 1-2 (Apr.-May), 1-8.

Paul, W. J. 1984. On heads versus tapes. Theoret. Comput. Sci. 28, 1-2 (Jan.), 1-12.

PaUl, W. J., SeIferas, J. I., AND Simon, J. 1981. An information-theoretic approach to time bounds for on-line computation. J. Comput. Syst. Sci. 23, 2 (Oct.), 108-126.

RABIN, M. O. 1963. Real time computation. Is. J. Math. 1, 4 (Dec.), 203-211.

SCHNITZleIN, W., AND STOB, H.-J, 1989. Linear-time simulation of multihead Turing machines. Inf. Comput. 81, 3 (June), 353-363.

STOß, H.-J. $1970 . \quad k$-Band-Simulation von $k$-Kopf-Turing-Maschinen. Computing 6, 3, 309-317 (in German).

VALIEV, K. 1970/1973. Certain estimates of the time of computation on Turing machines with an input. Cybernetics 6, 6 (June 1973), 734-741 (translated from Russian, published in Kibernetika 6, 6 (Nov.-Dec. 1970), 26-32). 
VITÁNyı, P. M. B. 1984. On two-tape real-time computation and queues. J. Comput. Syst. Sci. 29, 3 (Dec.), 303-311.

Zvonkin, A. K., AND Levin, L. A. 1970. The complexity of finite objects and the development of the concepts of information and randomness by means of the theory of algorithms. Russ. Math. Surv. 25, 6 (Nov.-Dec.), 83-124.

RECEIVED SEPTEMBER 1995; REVISED FEBRUARY 1997; ACCEPTED NOVEMBER 1996 